\title{
Performance of a Contrarotating Small Wind Energy Converter
}

\author{
Riadh W. Y. Habash, ${ }^{1}$ Voicu Groza, ${ }^{1}$ Yue Yang, ${ }^{2}$ Charles Blouin, ${ }^{2}$ and Pierre Guillemette ${ }^{3}$ \\ ${ }^{1}$ School of Electrical Engineering and Computer Science, University of Ottawa, Ottawa, ON, Canada K1N 6N5 \\ ${ }^{2}$ Department of Mechanical Engineering, University of Ottawa, Ottawa, ON, Canada K1N 6N5 \\ ${ }^{3}$ TRIAS Innovations Group, Ottawa, ON, Canada K1L 8K4 \\ Correspondence should be addressed to Riadh W. Y. Habash, rhabash@site.uottawa.ca
}

Received 25 January 2011; Accepted 21 March 2011

Academic Editors: D. Aggelis and M. Yürüsoy

Copyright ( $) 2011$ Riadh W. Y. Habash et al. This is an open access article distributed under the Creative Commons Attribution License, which permits unrestricted use, distribution, and reproduction in any medium, provided the original work is properly cited.

\begin{abstract}
Responding to more demand in coming years, the task of the small wind energy industry requires progress on several fronts-the public policy initiatives, technology development, and market growth. One important issue of the wind energy utilization is the conversion efficiency of the usable energy into productive power. Enhanced technologies such as contrarotating blades, gearbox and lubrication, airfoils, generators, and power electronics will lower cost and increase energy production. The purpose of this paper is, therefore, to reinforce the effectiveness of employing contrarotating system to enhance the performance of a small wind energy converter (SWEC). With this concept, the SWEC works more efficiently and therefore produces more energy in a unit turbine area. To verify the SWEC performance, a research model has been built and tested over a range of operating conditions. Wind tunnel tests were carried out to ascertain the overall performance of the contrarotating SWEC. Results are presented for cases of different wind speeds and Reynolds number. The results demonstrated a significant increase in wind energy conversion efficiency and capability of operation at lower wind speeds while keeping up performance compared to a single-rotor system of the same type.
\end{abstract}

\section{Introduction}

Wind energy has been shown to be one of the most feasible sources of renewable energy. It presents attractive opportunities to a wide range of people, including investors and entrepreneurs. The main goal of wind energy industry is to minimize the cost of wind energy in order to make it more competitive compared to other energy sources. How to reduce the cost of wind energy is a vital engineering challenge presented by the interlocking disciplines of aerodynamics, structure, control, electrical conversion, and electronics. In fact, technologies in these related areas are still under active research and development to achieve high efficiency and low cost.

In the shadows of advancing multimegawatt wind turbines is another growing sector within this industry, the small wind turbines. Small wind energy converters (SWECs) for urban or rural applications range in size from a few hundred watts to thousands of watts (usually with a rated capacity of less than $100 \mathrm{~kW}$ ) and can be applied economically for a variety of power demands. These systems can be used in connection with an electricity transmission and distribution system, or in stand-alone applications that are not connected to the utility grid and are appropriate for homes, farms, or even entire communities. Investments in this sector are feasible not as stand-alone only, but as components of an integrated power-generating system that include various forms of energy resources. The main technical challenges for SWECs are the design of a system that has maximum efficiency in turbulent low speed winds; ability to comply with both efficiency expectations and the requirements of grid utilities; and have the minimum environmental and health impacts in terms of noise and vibration.

Two facts characterize the urban environment for wind energy: lower annual mean wind speed (AMWS) compared to rural areas or to sea shores and more turbulent flow. The low AMWS is related to the uneven ground created by buildings and other features of the urban landscape, which causes wind speeds to increase with height above the ground 
more slowly. The turbulent flow is a result of the wind interacting with landscape obstacles, the fact that applies extra stress on the turbine blades. The challenge is to develop wind turbines that operate at lower speeds and cope with the turbulent.

The wind generating technology development is leading to improved performance and efficiency. Most wind turbines are single-rotor systems, which provide simplicity, reliability, and durability. Along the years, improvements have enhanced energy conversion efficiency of these singlerotor systems. For example, blades have better aerodynamic characteristics, gears with reduced noise have better torque transmission efficiency, and alternators have better electrical efficiency. However, despite these improvements, singlerotor systems are able to convert only a small fraction of the total wind stream energy into electrical energy. Moreover, such a system requires high wind velocity (above $4 \mathrm{~m} / \mathrm{s}$ ) which is not available in many places, a part from costal regions. This low velocity and seasonal winds imply a high cost of exploitation of wind energy. Thus, the challenge lies with the design of a wind generator which can operate at lower speeds and be used in a small-scale manner in remote and rural areas.

This paper investigates the performance of the SWEC basing on wind tunnel tests. The paper is organized as follows. Section 2 discusses the contrarotating concepts and provides a literature review on the subject, while Section 3 presents the theory of rotor torque and power. Section 4 describes the wind tunnel experimental setup, and Section 5 presents the rotor performance results. Finally, conclusions are drawn in Section 6.

\section{Contrarotating Blade System}

The prime mover in wind energy system is the wind turbine. One prevailing trend in wind turbine technology throughout the past couple of decades has been growth in the size of the rotor to realize the advantages of scale and the generally higher winds available at greater heights. Geometrically, consistent upscaling of blade length shows that the surface stresses at the blade surface, vibratory loads, and loading noise due to aerodynamical and gravitational loads grow in proportion to the length of the blade [1]. However, an alternative mean of overcoming the limitation of the efficiency of a single-rotor system without increasing the size of the rotor and consequently the stress on blades could be through the adoption of a dual-rotor (contrarotating) blade system. In addition, the acceptance of wind turbines by the public depends strongly on achieving low noise levels in operation, which largely depends on the level of stress on the blades.

According to Betz theory, the maximum power that can be extracted from the wind is about $59 \%$ of the available energy in the wind when the axial wind speed is reduced by two-thirds across a single rotor disc. However, practical wind turbines convert less than $40 \%$ of the wind energy into electrical energy. Hence, nearly $60 \%$ of the potential wind energy escapes without being harnessed. In reality, the energy in the wake behind a single rotor is not very small. Part of this energy may be extracted further by installing a second rotor in the wake. As the wake behind the first rotor is rotating in the opposite direction to the rotational direction of the rotor, the second rotor should rotate in the same direction as the wake in order to extract efficiently the available energy in the wake.

The contrarotating system is a very old concept that was initially proposed more than 100 years ago. A friend of Betz who is sometimes described as the "father of modern wind energy collection theory," Hans Honneff, wrote a book on the use of contrarotation, using two rotors one behind the other, driving the two halves of an electrical generator, therefore creating a true wind turbine [2]. Currently, the contra concept is used on airplanes, boats, and submarines to increase efficiency while eliminating the asymmetrical torque faced by conventional rotors.

A dual-rotor system can be described as a system consisting of two rotors separated by an appropriate distance (Figure 1). One of the rotors is rotating in counterclockwise direction and the other in clockwise direction on the same axis. The relative size as well as the appropriate distance between the two rotors should be identified for best performance. Drawbacks of the dual-rotor system come from mechanical complexity based on the fact that in order to reverse direction of rotation of one rotor, a gearbox is required. This may increase weight or maintenance and spare parts cost for the system.

Based on evidence in literature, aerodynamic research is poised between experimental and computational: either the wind turbine is studied experimentally in a wind tunnel, or the turbine is investigated computationally using methods that belong to the field of computational fluid dynamics (CFD). The two are closely linked, and as progress is made in the development of more advanced computational fluid models, more comprehensive wind tunnel experimental data is required to validate the models. Experimental and computational research provide results for better understanding of the flow physics and enable investigation of wind energy performance, a requirement in order to adjust the design of wind turbines to the unique aerodynamic conditions in the environment. As with all methods of analysis, the CFD approach has limitations which are essentially related to turbulence modeling. Sumner et al. [3] review the development of CFD as a virtual, multiscale wind tunnel applied by the wind energy community from small to large scale. Although the cost of a CFD analysis may be comparable to that of a wind tunnel experiment, we considered the wind tunnel experimental option for the current study emphasizing on the importance of transition to turbulence effects. Typically, wind tunnel tests overstate performance, and consumers will never see the performance measured in a wind tunnel. However, such tests are good indicators of performance. To our knowledge, only a limited number of wind tunnel studies can be found in literature $[2,4]$. In order to study the streamlines and obtain the detailed information of flow around the wind turbine, a flow visualization and velocity measurement are important. Investigation [5] has been carried out for this sake. Considerable improvements in the 


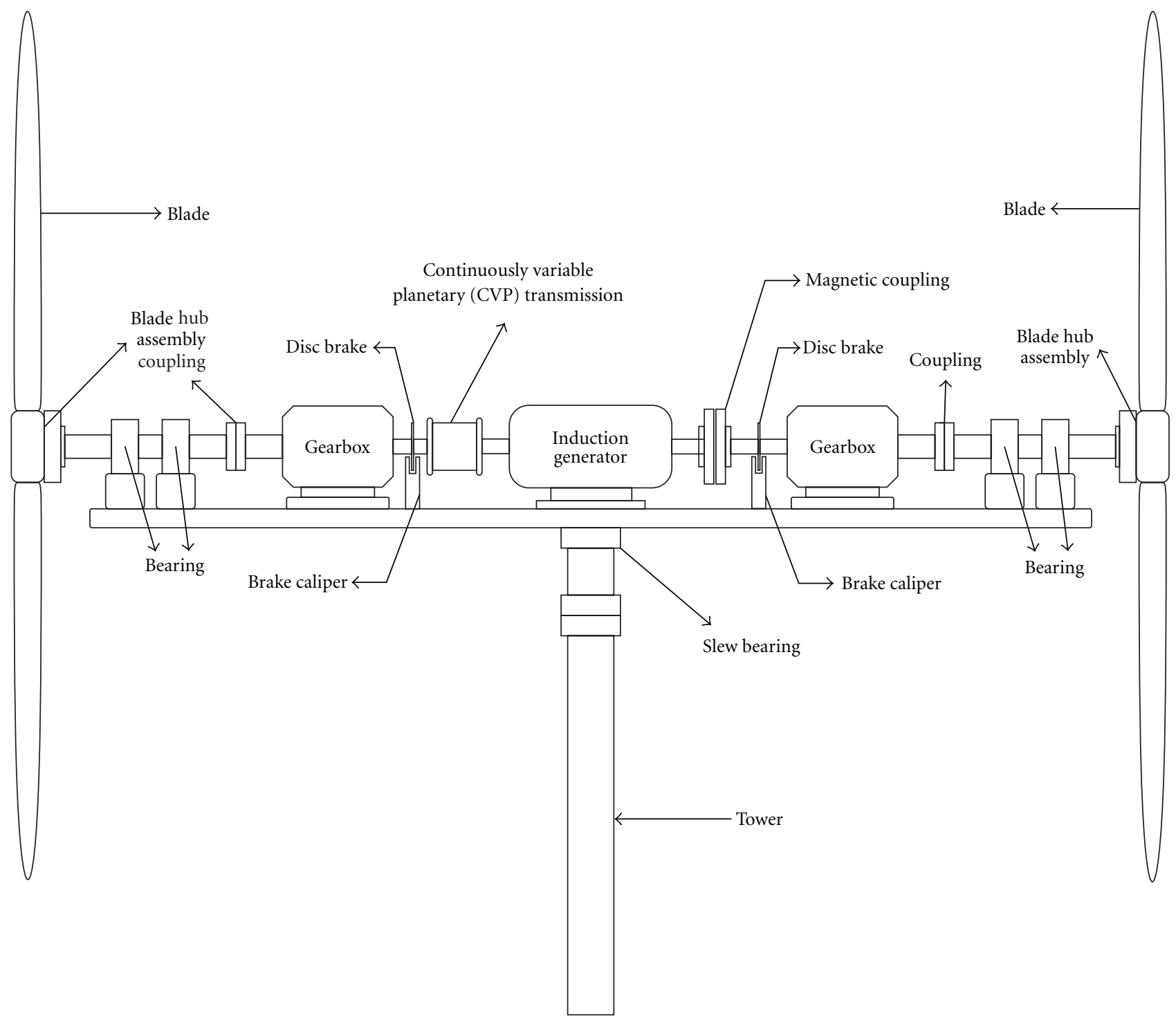

FIGURE 1: The proposed contrarotating small wind energy converter (SWEC).

understanding of contrarotating wind turbine system can be achieved through proper instrumentation and experimental measurements.

According to [6], the maximum power that can be extracted from a dual-rotor system increases up to $64 \%$ of the available energy. It continues to reach $66.7 \%$ for an infinite number of rotors [7]. A contrarotating wind turbine equipped with two $500 \mathrm{~kW}$ turbines performed quite well at high wind speeds. The turbine can produce $43.5 \%$ more annual energy than a single-rotor turbine of the same type. The performance of the system can be improved if it is operated for low wind speeds at the tip-speed ratio where a maximum Cp is obtained [8].

Research studies provide sufficient evidence to look closer at the concept of contrarotating system to eventually produce quantifiable comparisons to other turbines $[9,10]$. A smaller gear ratio is needed because of higher tip speeds achieved by smaller blade length in comparison with the conventional system in case of the same power output. Energy capture in the rotor holds the greatest potential for long-term reduction of the cost of wind energy.

A feasibility study [11] provides sufficient evidence to look closer at the concept of contrarotating to eventually produce quantifiable comparisons to other turbines. Their field tests showed that a dual-rotor turbine produces $43.5 \%$ more annual energy than a single-rotor turbine of the same type. In addition, a smaller gear ratio is needed because of higher tip speeds achieved by smaller blade length in comparison with the conventional system in case of the same power output [12]. According to a field test demonstrated in California [13], energy extraction from a wind turbine using contrarotating system increased by up to $40 \%$ over an equivalent wind turbine with only one rotor. Power conversion efficiency was high at low rotor speeds, suggesting applicability of contrarotating turbines to large utility-scale wind turbines that rotate at $16-20 \mathrm{rpm}$. In addition, the 
bending stress on the supporting tower was reduced by the contrarotating system over the single-rotor system. This reduced bending stress results when the torques produced by two rotors counterbalance each other.

The contrarotating SWEC clearly has a promise for wind energy, and after preliminary research and field studies [613], it was decided to proceed with a small SWEC prototype for testing and evaluation.

\section{Rotor Torque and Power}

The motion of any fluid can be derived from the basic physical principles of mass, momentum, and energy interchange. The torque responsible for power production of the wind turbine mostly arises due to the forces produced by interaction of blades with the wind. The output power $P_{T}$ from a turbine rotor and the wind kinetic energy per unit time $P_{W}$ are given as follows:

$$
\begin{gathered}
P_{T}=T_{m} \times \omega, \\
P_{W}=\frac{1}{2} \rho \times V_{0}^{3} \times A,
\end{gathered}
$$

where $T_{m}$ is the mechanical torque at the turbine side, $\omega$ is the angular rotation of the shaft, $\rho$ is the air density at the hub height, $V_{0}$ is the wind velocity, and $A$ is the swept area of the blades. If momentum equation is solved across an idealized control volume about the turbine rotor, it can be shown that the percentage of the total power available that can be extracted by a turbine is $16 / 27$ or $59 \%$. This limit is known as the Betz limit. Therefore, the maximum power that a turbine can produce is expressed as follows [14]:

$$
P_{W}=\left(\frac{16}{27}\right)\left(\frac{1}{2}\right) \rho \times V_{0}^{3} \times A .
$$

Most turbines extract the maximum possible energy as defined above for lower wind speeds but gradually become less efficient as the on-coming wind speed increases and the flow condition across the blades approaches the stall condition.

The rotor power coefficient $C_{p}$ is defined as the ratio between the rotor output power and the dynamic power of the air as shown in the following:

$$
C_{p}=\frac{P_{T}}{P_{W}}=\frac{T_{m} \times \omega}{(1 / 2)\left(\rho \times V_{0}^{3} \times A\right)} .
$$

The power coefficient is a nonlinear function of the tip speed-ratio $\lambda$, which depends on the wind velocity and the rotation speed of the shaft

$$
\lambda=\frac{V_{\text {Tip }}}{V_{0}}=\frac{r \times \omega}{V_{0}},
$$

where $r$ is the rotor radius. The rotor power coefficient is regarded as the energy transformation efficiency. Note that $C_{p}$ is usually precomputed based on the theoretically expected performance of the turbine system.

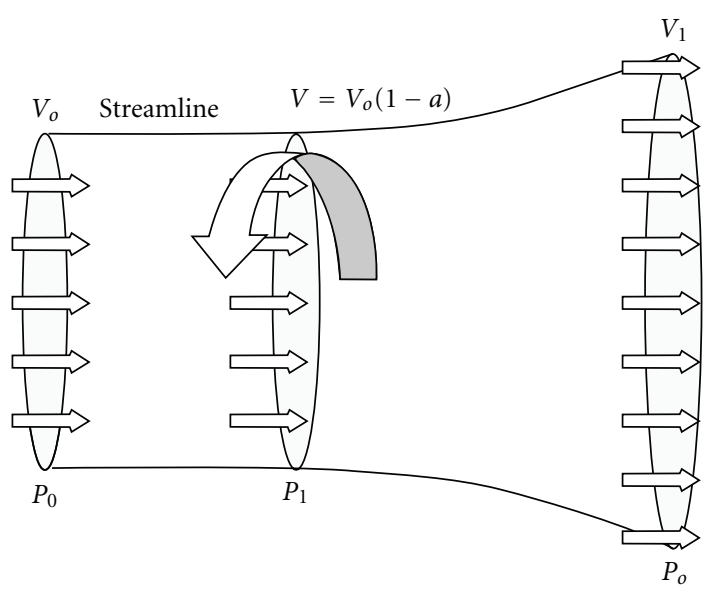

FIGURE 2: Stream tube passing the rotor plane.

The wind turbine mechanical characteristics are described by the following equation (where the turbine rotor friction is ignored):

$$
T_{m}-T_{g}=J \frac{d \omega}{d t}
$$

where $T_{g}$ is the load torque, and $J$ is the turbine inertia moment.

The incoming wind flow rate should be equal to the outgoing flow rate to satisfy the mass conservation law if a control volume around a turbine is assumed. The outgoing wind-speed distribution and its direction strongly determine the turbine efficiency. Figure 2 shows the geometry of the stream tube through the disk. Neglecting fluid drags, the power extracted from the air stream can be written as

$$
P=\frac{1}{2} \rho A V\left(V_{o}+V_{1}\right)\left(V_{o}-V_{1}\right)
$$

where $V, V_{o}$, and $V_{1}$ are the flow velocity components along the axis of the stream tube. The power coefficient is obtained by nondimensionalizing the above power equation as

$$
C_{p}=\frac{P}{0.5 \rho V_{o}^{3} A}=4 a(1-a)^{2},
$$

where $a$ is the axial induction factor.

\section{Wind Tunnel Experimental Setup}

In this section, laboratory measurement techniques are discussed; however, some of the methods used are conventional and require little elaboration.

4.1. Wind Tunnel Facility. An open-return type wind tunnel is used in the present study. A contrarotating model 3-blade wind turbine was placed in the boundary-layer wind tunnel with the goal of studying power performance, turbulence effect, and flow visualization. Figure 3 shows the schematic of the wind tunnel experimental setup where the contraction and test sections are on the right hand side, and the motor 


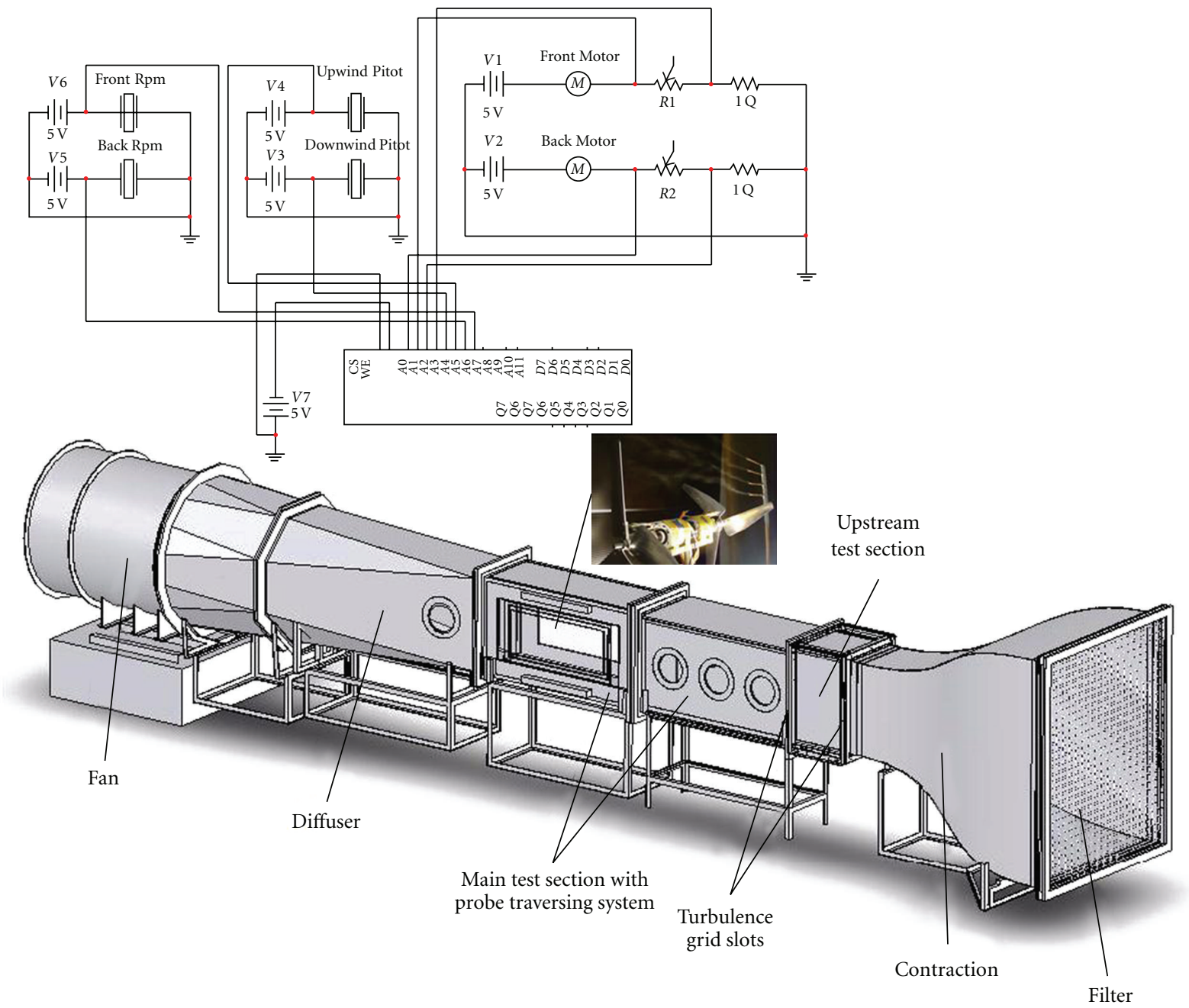

(a)

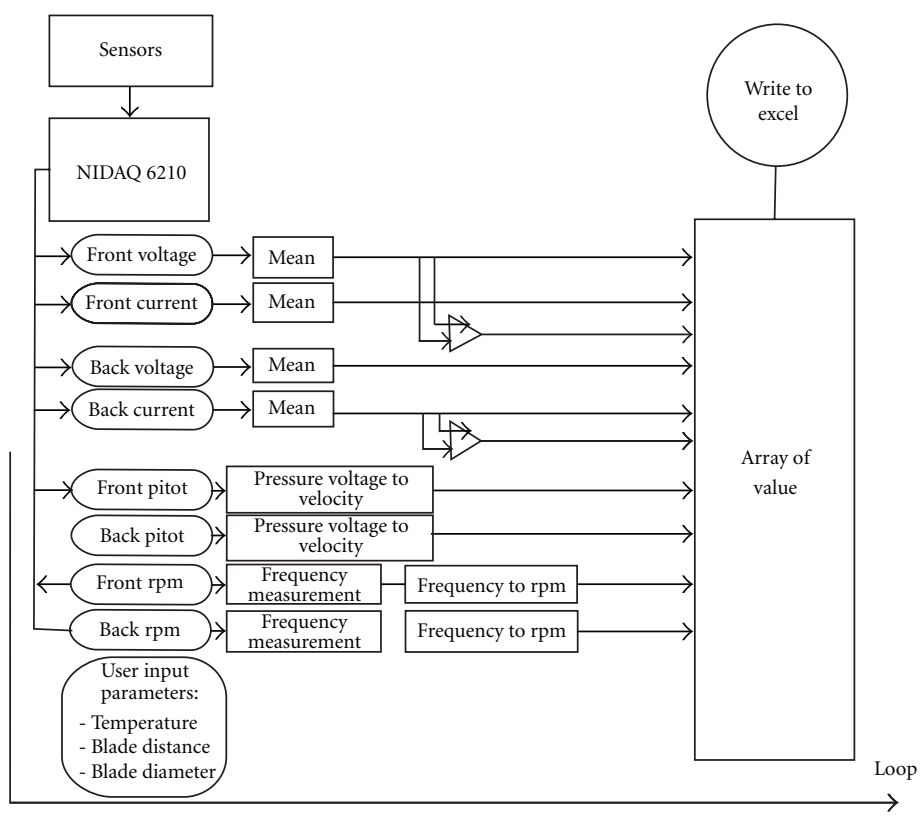

(b)

FIGURE 3: Wind tunnel test facility. (a) Electrical part of the setup. (b) Instrumentation setup via LabView. 
and fan are in the left hand side. Air enters the fan from the laboratory through a large gate covered by a filter, held by wire meshes. The air flow is driven by a propulsion system made of an axial fan to provide the dynamic pressure for compensating viscous losses. There are smooth glass walls on both sides of the tunnel, and access is possible through the plywood ceiling and floor. Any large obstruction placed within a wind tunnel will alter the characteristics of the flow to some degree. The wind tunnel is capable of generating wind speeds up to $30 \mathrm{~m} / \mathrm{s}$. This suction type wind tunnel has a cross-section of $0.61 \mathrm{~m}$ width by $0.9 \mathrm{~m}$ height. The tunnel has a working (test) section of length $3.6 \mathrm{~m}$. As the test section is the narrowest part of the circuit, it is also the part where the air velocity is the highest and, therefore, by Bernoulli's principle, where the pressure is the lowest. The main distinguishing feature of this wind tunnel is that it was designed to produce a low level of turbulence in the test section. Power for the tunnel comes from a three-phase AC motor of $30 \mathrm{hp}$ at $1800 \mathrm{rpm}$ with a maximum speed of $1170 \mathrm{rpm}$, driving a 10-bladed fan of 54 inches diameter with blade setting of $23^{\circ}$, mounted in a cylindrical steel casing. To minimise noise and vibration, the casing is supported on rubber shock mounts and is connected by flexible seals to the tunnel on either side. The air speed does not change as the air passes through the fan. The rotational speed of the fan is controlled by a regulated magnetic field and solid-state power supply.

In order to control the ambient turbulence level, turbulence manipulators are placed upstream of the rotor, including a fine mesh screen and an aluminum honeycomb section. Smoothing is provided by the fine mesh screen. The honeycomb plays the role of a flow straightener. When the wind turbine is stopped, the mean velocity over the center portion of the wind tunnel is uniform and almost steady.

4.2. Instrumentation. A small model SWEC with two blade sets of $23 \mathrm{~cm}$ diameter and a varying distance between the blade sets of $7-54 \mathrm{~cm}$ has been built and tested over a range of operating conditions. In order to introduce some degree of uniformity into the way in which users of the wind tunnel record their data, an instrumentation system to measure and display a number of variables that are normally required for all experiments was installed. Two guide rails were used to hold the SWEC inside wind tunnel floor along the centreline using a steel mounting system. The steel mounting system ensured that the system did not move during testing. Measuring sensors were mounted at different locations of the setup. The upwind and downwind velocities are measured by pitot tubes, which use Bernoulli's principle to convert pressure to velocity readings. The tubes are attached to 2 sensors to convert pressure in volts to velocities in $\mathrm{m} / \mathrm{s}$. For measuring the rotational speed of the rotor, two infrared detector and emitter units (photogate sensors) were used. They were mounted behind the rotor.

Measurement depends largely on a data acquisition system utilizing electronic measuring to read instantaneous power produced by the generating system, as various parameters are varied on the turbine or in the environment. The parameters varied include the distance between the two sets of blades, blade profiles, number of blades, wind speeds, and size ratios. To accomplish the objective of this test, three aspects of experimental setup are needed: mechanical, electrical, and measurement software.

All sensors are powered, grounded, and connected to the data acquisition board. All wires are shielded for protection against noise. Measurements are monitored directly and instantaneously in the Graphical User Interface (GUI) of LabView. The user enters numerical values of the blade distance, blade pitch, and blade diameter for the front and back and the relative humidity and temperature. The circuit has 5 sets of measurements on both the front and the back of the generating system. The voltages are measured directly from the potentiometers; these are the total voltages of the circuits. The currents are obtained by measuring the voltages from fixed resistors and dividing that by the resistance. The power is the product of the voltage and the calculated current. The rpm signals go through a frequency measurement tool in LabView and are then multiplied by 60 to obtain the angular velocity in revolutions per minute (rpm). All lines of measurements are connected to the National Instruments Data Acquisition Board NIDAQ USB6210. Each line is connected to an analog pin which is fed into the LabView program with a USB connection to the computer.

At the beginning of the measurement process, all sensors were checked and calibrated. The pitot tubes are corrected by the offset values to give zero when there is no wind in the tunnel. When starting the program, a path is requested for an Excel file to record the data.

\section{Rotor Performance Tests}

5.1. Energy Conversion Test. The main factor which characterizes the wind turbine is its power curve, which is defined as a table of data, consisting of connected values of net electric power from the wind turbine and the wind speed. The net electric power from the electric generator is calculated using loss separation

$$
P_{\text {net }}=P_{\text {output }}+P_{\text {ohmic }}+P_{\text {core }}+P_{\text {friction }} .
$$

The output power $\left(P_{\text {output }}\right)$ is measured using a power analyser. The analyser determines the power, electrical frequency, and stator currents from the generator to a variable resistive load bank. The load bank is used to vary the load on the generator, at a particular incident wind speed, in order to determine the maximum power point. The net power $\left(P_{\text {net }}\right)$ is then determined by incorporating resistive losses $\left(P_{\text {ohmic }}\right)$ associated with power loss in the stator resistance, core loss $\left(P_{\text {core }}\right)$ associated with hysteresis and eddy-current losses in the iron core of the machine, and friction and windage losses in the generator $\left(P_{\text {friction }}\right)$.

Choosing a rotor with the optimal aerodynamic characteristics is a significant step in the design of the SWEC. However, all this will be for nothing if the electrical side of the system is inadequately thoughtout. Based on the maximum speed expected for the SWEC and taking into consideration 


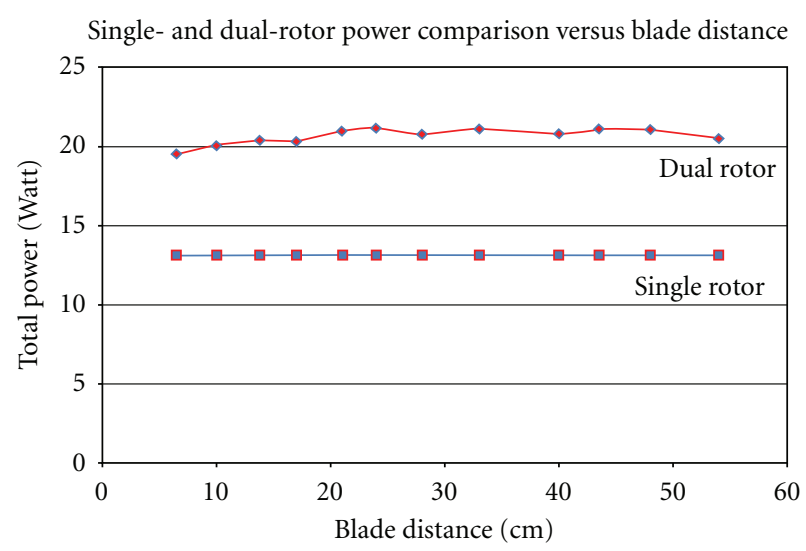

Figure 4: Comparison of power output between single- and contrarotating system.

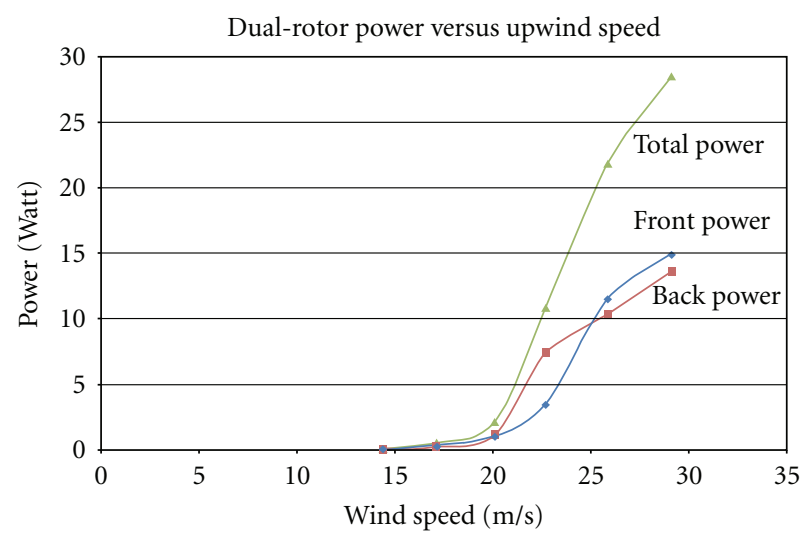

FIGURE 5: Effect of wind speed on output power.

the cubic relationship between the wind speed and the generated power, the designer must select the generator to match these requirements. The generator can be either fixed or variable speed. Fixed speed generators, like the synchronous machines, operate at speed dictated by the frequency of operation and the number of magnetic poles. Therefore, they are not ideal for wind systems because wind speed is variable. Variable speed generators include DC generators and induction generators. Of the two variable-speed generators, the DC generator is generally used for standalone systems, while the induction generator (squirrel cage and wound rotor) is most widely used in applications that supply electricity directly to the grid. For the experimental SWEC setup, two small laboratory-based permanent-magnet machines (18 V; $400 \mathrm{~W} ; 21000 \mathrm{rpm}$ ) were used as a generating unit.

Power production tests were conducted with a contrarotating blade system as well as single-rotor system. The testing procedure is conducted to experimentally determine power curves for each blade set and generator at various wind speeds. The maximum power for the corresponding wind speed was calculated using the power equation. For the single-rotor results, the other blade set of the SWEC prototype was taken off. Tests were conducted at the wind

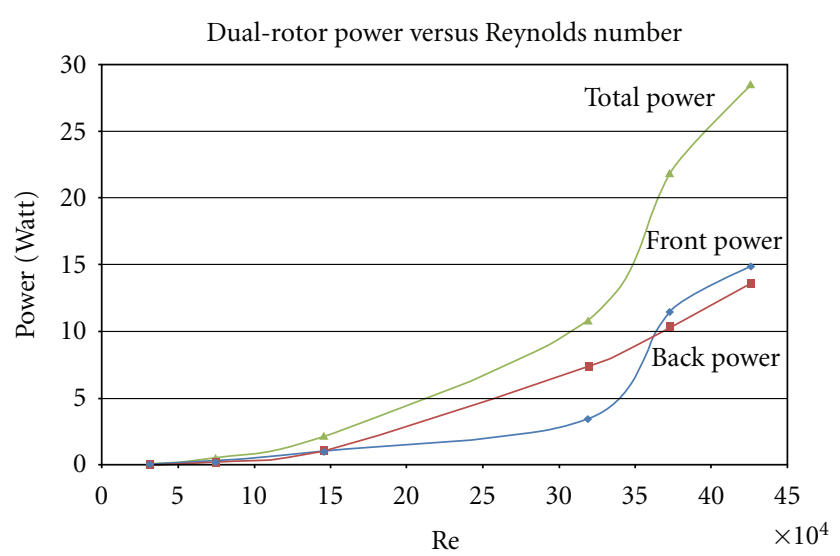

FIGURE 6: Effect of Reynolds number on output power.

tunnel by increasing the wind speed from 14 to $29 \mathrm{~m} / \mathrm{s}$ in increments of $3 \mathrm{~m} / \mathrm{s}$. To test the efficiency of these blades, the peak power extracted from the wind was taken for both sets of blades. Both blade sets produced approximately the same amount of power with the back blade set producing slightly less power than the front set at low and high wind speeds. The results of the measurements have produced the necessary data for the evaluation of the rotor blade performance.

In general, results of the wind tunnel test indicated that a scaled-down version of the contrarotating SWEC may produce up to $60 \%$ more energy than a single-rotor system of the same type (Figure 4). At lower speed, the contrarotating SWEC can extract more energy over an equivalent single-rotor SWEC. Test results showed that the SWEC under consideration could harness energy at reduced wind speeds while keeping up performance (Figure 5) and keep functioning and extracting energy at higher wind speeds (up to $35 \mathrm{~m} / \mathrm{s}$ ). Usually, during high wind gusts, conventional turbines turn away from the wind or shut down. For a special upwind speed interval of $20-25 \mathrm{~m} / \mathrm{s}$, there is a sudden jump in the back power (Figure 5), while the front continues to increase slightly. Then, the pattern changes with a sudden jump for the front blade. This may be attributed to the effect of turbulence.

Aerodynamic studies are available largely for flows with Reynolds number greater than $10^{6}$ (Reynolds number is approximately $\operatorname{Re}=\rho v D / \mu$, where $\rho$ is density, $v$ is velocity, $D$ is diameter of object, and $\mu$ is kinematic viscosity). However, there are not many computational or experimental studies available for flows at very low Reynolds number. Some experimental studies are reported $[15,16]$. Figure 6 compares the effect of Reynolds number on output power for single- and dual-rotor SWECs. At approximately $21 \mathrm{~m} / \mathrm{s}$ upwind speed, which is equivalent to a Reynolds number of 280000 , there is a sudden increase in the front blade power. This pattern coincides very closely with Figure 5. Due to the test section size of the wind tunnel, a similarity comparison between the SWEC prototype and a full-scale SWEC, based upon Reynolds number, is not possible. A valid alternative scaling technique must be determined. 


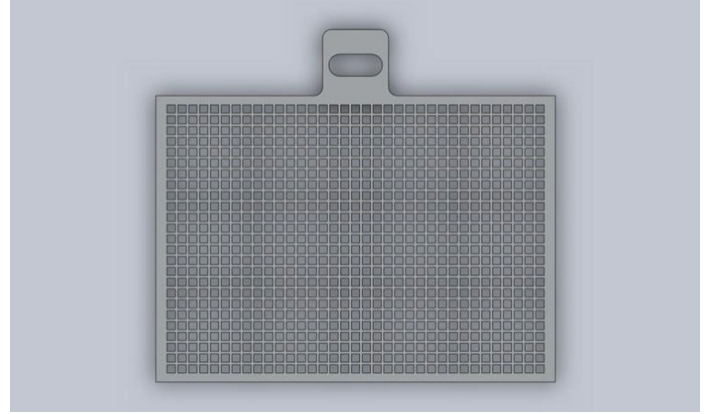

(a) Holes in the above screen are $19 \mathrm{~mm} \times 19 \mathrm{~mm}$ (small)

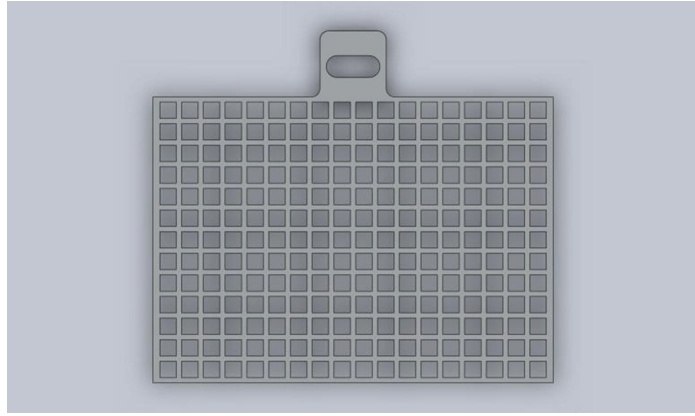

(b) Holes in the above screen are $38 \mathrm{~mm} \times 38 \mathrm{~mm}$ (medium)

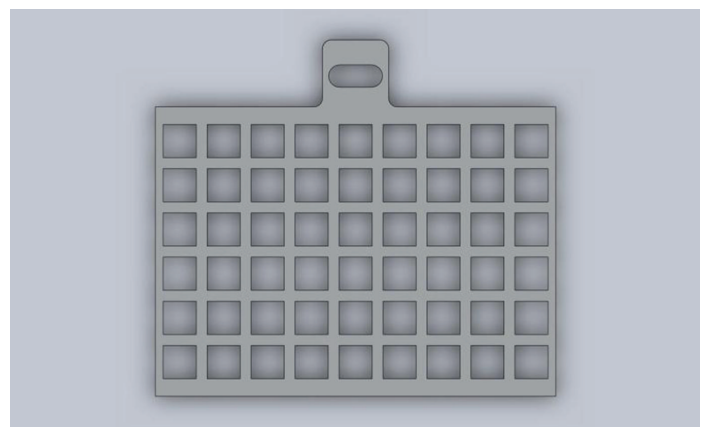

(c) Holes in the above screen are $76 \mathrm{~mm} \times 76 \mathrm{~mm}$ (large)

Figure 7: Three types of screens used in the wind tunnel.

5.2. Turbulence Test. Understanding of the turbulent properties of wind turbine wakes under different inflow and surface conditions is important to maximize the energy production and ensure the structural reliability of the wind turbines. The structure of wind turbines is known to be affected by the characteristics of the incoming flow. For example, Sheinman and Rosen [17] have shown that neglecting the effect of turbulence in the incoming flow can lead to an overestimation of turbine output by more than $10 \%$. Turbulence intensity $\left(I_{u}\right)$ plays a direct role on the mean force $(F)$ and bending moment $(M)$ acting on the turbine [18] and is commonly defined as the standard deviation of the wind velocity component in the average wind direction $\left(\sigma_{u}\right)$ divided by the wind velocity at the turbine hub height $\left(U_{\text {hub }}\right)$

$$
I_{u}=\frac{\sigma_{u}}{U_{\text {hub }}} .
$$

Unwanted turbulence is usually generated in the wind tunnel at the wall (shear stress), at bends of the tunnel (secondary flows), on the model (vortex shedding), and by the fan. To minimize unwanted turbulence, walls should be smooth and carefully sealed, sudden changes in geometry should be avoided, and contraction before the measuring section may increase the mean velocity and decrease the relative turbulence. The most important feature for minimising the turbulence level is the contraction ratio, that is, the ratio of the cross-sectional areas at the wide and narrow ends of the contraction.

Experimentally, we inserted three types of screens (Figure 7) to produce turbulence in order to observe the

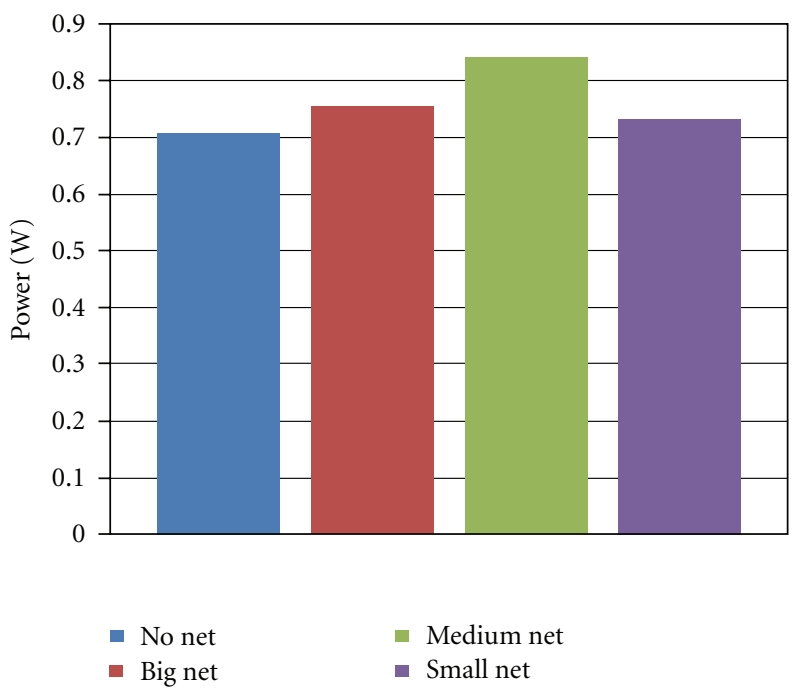

FIgURE 8: Output power as a function of screen type.

impact of turbulence on production of energy. It is evident from Figure 8 that there is some impact of screens on power production (some gain of up to $12 \%$ is noticed for the medium screen). However, we should take into consideration the limitation of the experiment in regard to controlling wind speed inside the test section of the wind tunnel.

5.3. Flow Visualization and Smoke. Flow visualization illustrates the flow on or near an object. It may provide important information on processes which are associated with many 


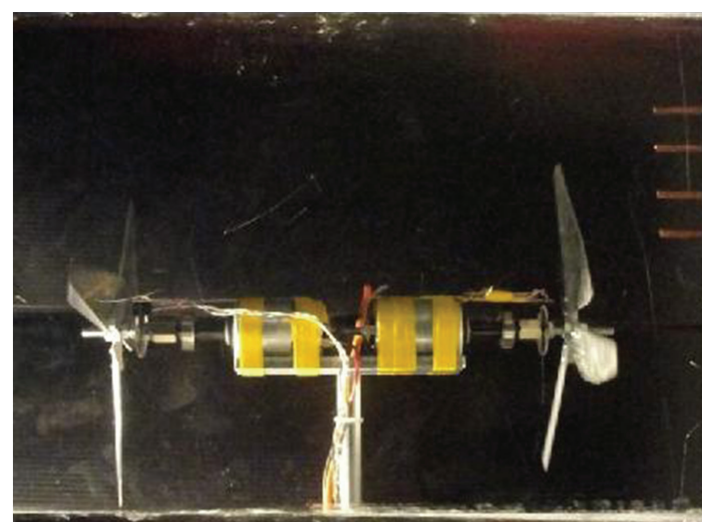

(a)

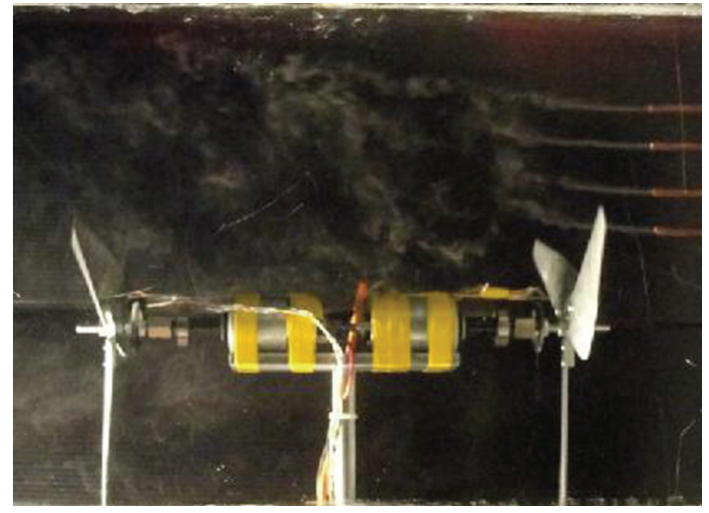

(c)

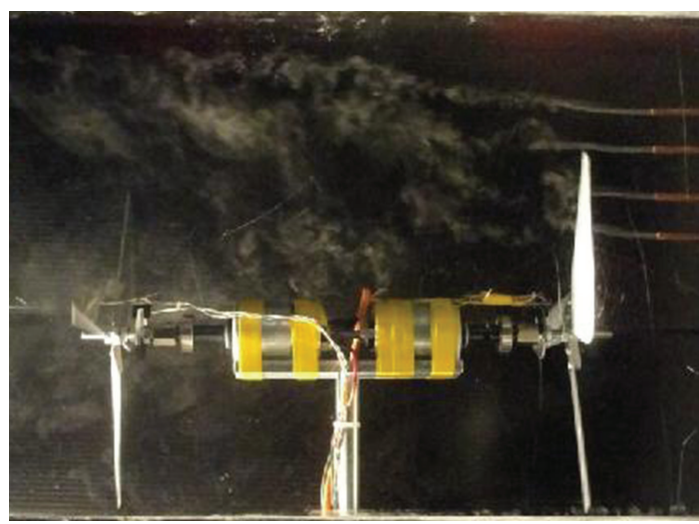

(b)

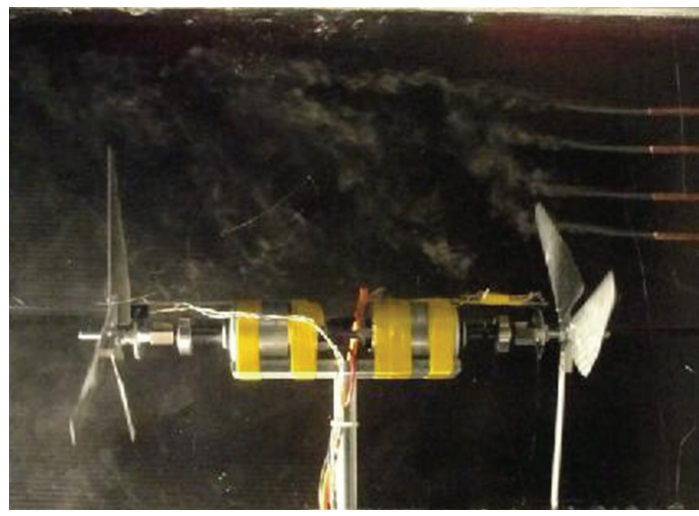

(d)

FIGURE 9: Four camera shots of smoke visualization of a contrarotating SWEC in wind tunnel.

problems of fluid flow. In gases, flow visualization is usually accomplished by injection of smoke. Various ways of producing smoke for this purpose have been developed. Although it is not difficult to develop a short-time duration smoke generator, certain problems are encountered when a longduration smoke of a constant production rate is required. Several techniques are available in literature; however, they cannot be used without "trial and error" approach [17].

The technique used in this investigation provides longtime duration smoke that forms a plane of closely spaced parallel streak lines. The developed smoke-generating system comprised of compressed air that is ducted through a jar containing a mixture of titanium tetrachloride and carbon tetrachloride and a smoke-dispensing rake. As a result of a chemical reaction and due to the presence of moisture in the air, a dense white smoke (titanium dioxide) is produced. The smoke is supplied through flexible tubing to a brass rake located in the wind tunnel. Smoke jets, approximately $0.0016 \mathrm{~m}$ in diameter, are generated downstream of the rake. A honeycomb is placed very close downstream from the rake in order to attenuate disturbances present in the streaklines of the generated smoke.

We used a high speed still-picture camera from HSI Inc. to monitor the fluid visualization in the turbulent wake and for the flow shape around the blades. A white, then black background was used for the back rectangular window, but the effects were not clear. Two $500 \mathrm{~W}$ lights with attached reflectors were placed behind the camera, projecting light into the prototype setup, behind the plexiglass of the tunnel window.

Figure 9 composed of four photos shows the information that can be obtained from smoke visualization of contrarotating blades in a wind tunnel at low Reynolds number. The top left picture is the setup without smoke. The flow goes from right to left in all pictures. It is evident that holes in the smoke patterns behind the right rotor are caused by the turning blades. The general shape of the flow around the wind turbine, represented by an expanding cylinder around the rotors, can clearly be observed.

The flow visualization technique described above proved to be effective for qualitative analysis of flow situations. The contrarotating system provides long-time duration, almost stable smoke streaklines, and is easy to operate and maintain.

\section{Conclusion}

Combining several or all of the advancements in wind energy technologies makes wind energy systems more efficient; capable to operate at lower wind speeds and keeping up performance, and quieter with gears of reduced noise and better 
torque transmission efficiency. The following conclusions emerge from this study.

(1) The literature indicates that a contrarotating wind energy system could extract additional 20-40\% power compared to a single-rotor system from the same wind stream. In this paper, the dependence of the aerodynamic performance of the SWEC on rotor system geometry has been studied by a small-scale model experiment in the wind tunnel. Test results indicate that a contrarotating system may produce up to $60 \%$ more energy than a single-rotor system of the same type and is capable to reduce cut-in speed while keeping up performance.

(2) In addition to energy conversion efficiency, the main problem that the contrarotating concept solves is improved performance in low-wind conditions.

Although the SWEC under development has great potential, it still needs further studies, including the design of blade construction; the optimization between the rotor and electric generator; power efficiency, power quality, and power factor; and the static and transient stability of the system. In future, the percentage of wind energy on many grids is expected to be a significant part, especially from SWECs thus making them as key grid players. However, these machines still require the best technologies from various disciplines to behave like power plants. The success of SWECs depends strongly on their ability to comply with both efficiency expectations and the requirements of grid utilities. An introductory video about the SWEC under development is available [19].

\section{Acknowledgments}

Financial support from the University of Ottawa and Ontario Centres of Excellence (OCE) is gratefully acknowledged. Advice and suggestions during the measurement program and data analysis activities from Professor Stavros Tavoularis of the University of Ottawa's Department of Mechanical Engineering are appreciated.

\section{References}

[1] C. Rajendran and G. Madhu, "A CFD based multibody dynamics approach in horizontal axis wind turbine," International Journal of Dynamics of Fluids, vol. 6, no. 2, pp. 219-230, 2010.

[2] I. Grant, M. Mo, X. Pan et al., "Experimental and numerical study of the vortex filaments in the wake of an operational, horizontal-axis, wind turbine," Journal of Wind Engineering and Industrial Aerodynamics, vol. 85, no. 2, pp. 177-189, 2000.

[3] J. Sumner, C. S. Watters, and C. Masson, "CFD in wind energy: the virtual, multiscale wind tunnel," Energies, vol. 3, no. 5, pp. 989-1013, 2010.

[4] L. J. Vermeera, J. N. Sorensenb, and A. Crespo, "Wind turbine wake aerodynamics," Progress in Aerospace Sciences, vol. 39, no. 6-7, pp. 467-510, 2003.

[5] P. R. Ebert and D. H. Wood, "The near wake of a model horizontal-axis wind turbine-I. Experimental arrangements and initial results," Renewable Energy, vol. 12, no. 3, pp. 225243, 1997.

[6] B. G. Newman, "Actuator-disc theory for vertical-axis wind turbines," Journal of Wind Engineering and Industrial Aerodynamics, vol. 15, no. 1-3, pp. 347-355, 1983.

[7] B. G. Newman, "Multiple actuator-disc theory for wind turbines," Journal of Wind Engineering and Industrial Aerodynamics, vol. 24, no. 3, pp. 215-225, 1986.

[8] W. Z. Shen, V. A. K. Zakkam, J. N. Sørensen, and K. Appa, "Analysis of counter-rotating wind turbines," Journal of Physics: Conference Series, vol. 75, no. 1, Article ID 012003, 9 pages, 2007.

[9] S. N. Jung, T. S. No, and K. W. Ryu, "Aerodynamic performance prediction of a $30 \mathrm{~kW}$ counter-rotating wind turbine system," Renewable Energy, vol. 30, no. 5, pp. 631-644, 2005.

[10] J. D. Booker, P. H. Mellor, R. Wrobel, and D. Drury, "A compact, high efficiency contrarotating generator suitable for wind turbines in the urban environment," Renewable Energy, vol. 35, no. 9, pp. 2027-2033, 2010.

[11] M. Czajkowski, Feasibility of a unique wind powered home heating system, Honors thesis, Mechanical Engineering Class, Potsdam, Germany, 2008.

[12] T. S. No, J. E. Kim, J. H. Moon, and S. J. Kim, "Modeling, control, and simulation of dual rotor wind turbine generator system," Renewable Energy, vol. 34, no. 10, pp. 2124-2132, 2009.

[13] H. Clark, Counter Rotating Wind Turbine System, California Energy Commission, 2003.

[14] R. S. Amano and R. J. Malloy, "CFD analysis on aerodynamic design optimization of wind turbine rotor blades," World Academy of Science, Engineering and Technology, vol. 60, pp. 71-75, 2009.

[15] S. Sunada, A. Sakaguchi, and K. Kawachi, "Airfoil section characteristics at a low Reynolds number," Journal of Fluids Engineering, vol. 119, no. 1, pp. 129-135, 1997.

[16] S. Sunada, T. Yasuda, K. Yasuda, and K. Kawachi, "Comparison of wing characteristics at an ultralow reynolds number," Journal of Aircraft, vol. 39, no. 2, pp. 331-338, 2002.

[17] Y. Sheinman and A. Rosen, "A dynamic model of the influence of turbulence on the power output of a wind turbine," Journal of Wind Engineering and Industrial Aerodynamics, vol. 39, no. 1-3, pp. 329-341, 1992.

[18] W. Merzkirch, Flow Visualization, Academic Press, New York, NY, USA, 1974.

[19] http://www.youtube.com/watch?v=wlf92In-kfk\&feature=player_embedded, 2011. 

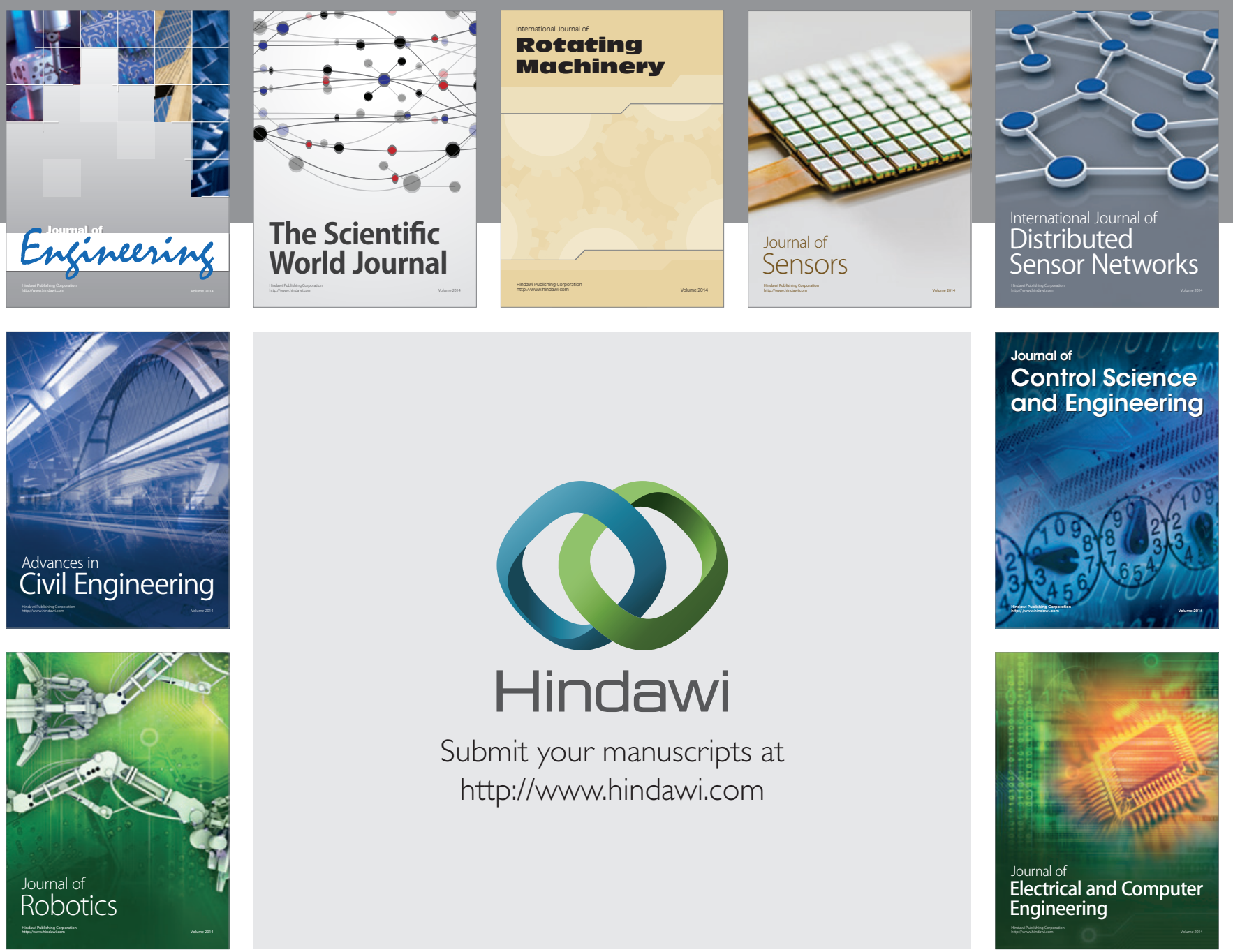

Submit your manuscripts at

http://www.hindawi.com
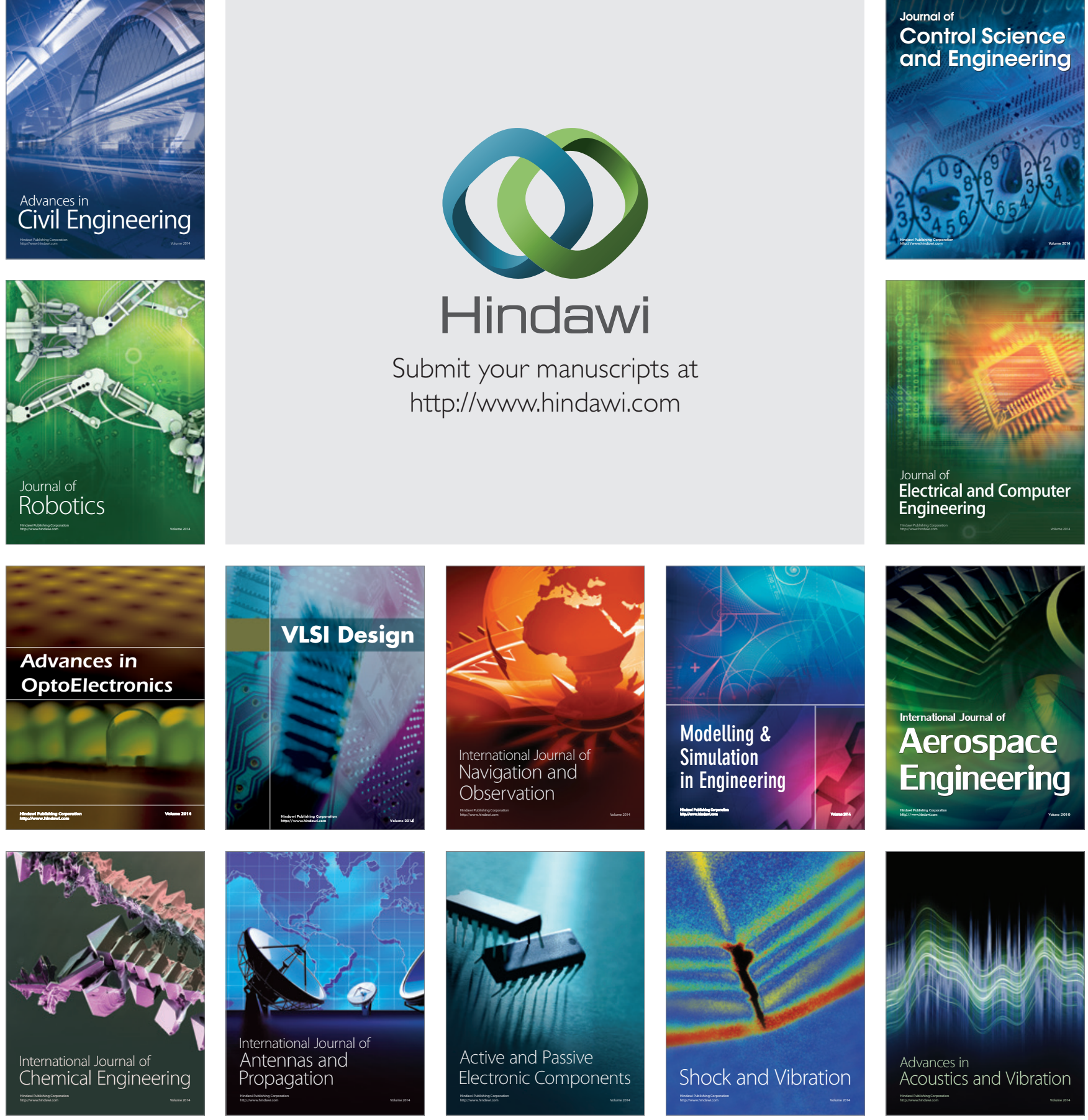